\title{
A STATISTICAL STUDY OF PAPERS IN THE JOURNAL OF BONE AND JOINT SURGERY [BR] 1984
}

RICHARD W. MORRIS

\author{
From St Thomas' Hospital, London
}

\begin{abstract}
The statistical quality of 103 original articles published in The Journal of Bone and Joint Surgery (British Volume) in 1984 was assessed. Some papers were found to be deficient: thus, it was not always clear how series were selected and sometimes neither the data nor the results were clearly presented. Sample sizes were frequently inadequate for the conclusions reached and statistical techniques should have been used more frequently. A majority of papers were descriptions of case series for which no comparative data were made available.

It is suggested that collaborative research would best advance knowledge about the relative benefits of various managements, and that statistical advice could make a substantial contribution.
\end{abstract}

Much debate has taken place in recent years about the quality of statistics in medical journals (Gore and Altman 1982); this has generally been judged poor and various remedies have been suggested, including improved education for doctors, more co-operation with statisticians and the statistical refereeing of more papers. The Editor of The British Volume of The Journal of Bone and Joint Surgery asked the author of this paper to review statistical aspects of the papers published in 1984 .

\section{METHOD OF REVIEW}

Various checklists have been proposed for the statistics in medical papers (Evans and Pollock 1985; Bland et al. 1985; Gardner, Machin and Campbell 1986) and from these a list was made which suited The Journal of Bone and Joint Surgery. This was modified after a pilot trial on papers published in 1983 and is given in Table $\mathrm{I}$.

A total of 135 papers were published in 1984. Of these 32 were excluded as they were review articles or editorials, or because they involved five or less patients. The remaining 103 papers were assessed by the author. As a control, a random sample of 26 of these, stratified to include representative proportions of studies with various sample sizes, were assessed by two other medical statisticians and reviewed again by RWM.

\section{FINDINGS}

The frequency of the various possible assessments for the 103 papers is shown in Table II.

R. W. Morris, MSc, Lecturer in Medical Statistics

Department of Community Medicine, United Medical and Dental Schools, St Thomas' Hospital, London SEI 7EH, England.

(C) 1988 British Editorial Society of Bone and Joint Surgery $0301-620 \times / 88 / 2066 \$ 2.00$

J Bone Joint Surg [Br] 1988;70-B : 242-6.
General features. The aims of the study were properly described in all but four papers; these aims form a useful guide for the reader. The population being reported on was at least implied in 89 of the 103 papers. The crucial question is: "To what group of subjects do our results apply?" This was often unclear and was sometimes applied uncritically. It is necessarily vague when animal experiments are reported.

The sample of subjects reported on may not be truly representative of the implied population. The reader must make his judgement on the basis of appropriate data and discussion. Unfortunately, only 53 papers seemed to contain enough information on the source of the study sample. Many papers simply stated the number of patients available for analysis, without reporting time period, place, or how the group were selected. It is important to know whether these patients were a subset of a larger group, some of whom may not have been followed up. If this information is lacking, there must be suspicion that the sample may have been highly selected. A useful method is to provide basic data for the whole group (age, sex, characteristics of the operation) so that this can be compared with the same data for the sample of patients actually studied in detail.

In general treatment was well documented, in so far as a non-medically qualified statistician could judge: in the 79 papers where a detailed description seemed to be required, this was adequate in 76 . Clearly this is essential for surgeons who wish to perform new procedures. Scientific category. Green and Byar (1984) suggest that there are eight categories of evidence by which the efficacy of treatment can be assessed. These range from anecdotal case reports to confirmed randomised controlled trials. A modified list of categories was applied (see Table I). No less than 73 papers reported case series without controls. In many of these reference was made to 
Table I. Checklist for assessing statistical aspects of papers

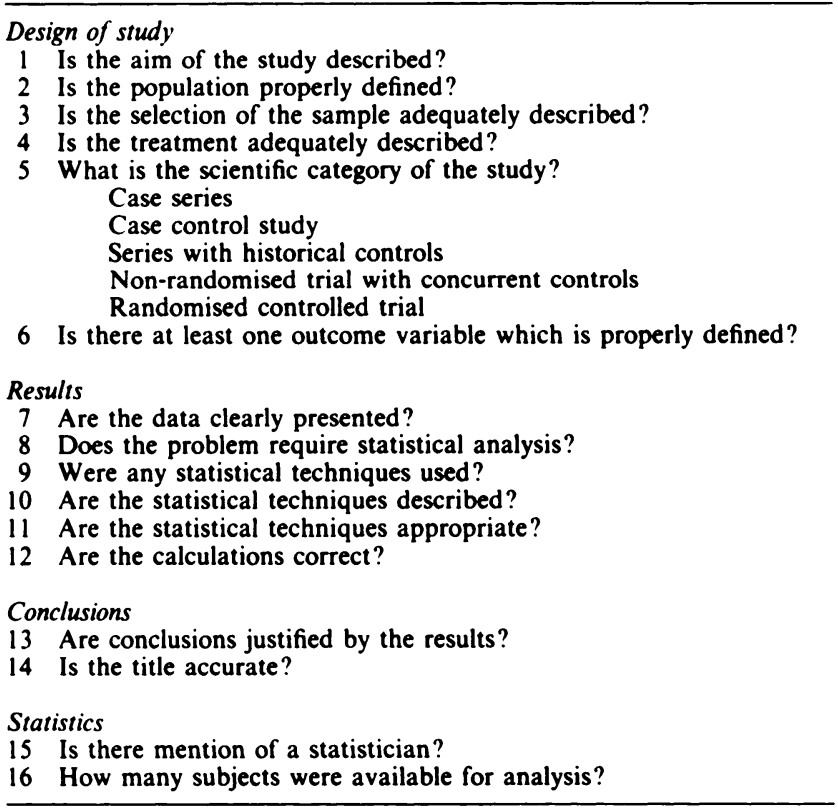

other published series, but details of important differences in patient mix, method of assessment, type of care, and length of follow-up were rarely provided.

A good example of this problem was seen in a report on the management of congenital dislocation of the hip: there were so many variables in selection, management and assessment that, lacking a common protocol, comparisons with other series had doubtful validity. Only five papers in 1984 reported randomised controlled trials. There were nine papers involving animal or cadaver experiments, community surveys or diagnostic technology.

Outcome variables. Taking an operation as a scientific experiment, a particular criterion or "end point" is required to assess its success; this should be as unambiguous as possible. Without such an end point no objective value can be set on the surgical procedure. It seemed that only 68 papers had at least one properly defined outcome variable.

To report outcome as "patients have been extremely pleased" is to neglect the need for quantitative data

Table II. Results of assessment of the 103 papers

\begin{tabular}{|c|c|c|c|c|c|c|}
\hline & & & Yes & No & Unclear & Not applicable \\
\hline 1 & \multicolumn{2}{|c|}{ Aim described } & 99 & 4 & & \\
\hline 2 & \multicolumn{2}{|c|}{ Population defined } & 89 & 14 & & \\
\hline 3 & \multicolumn{2}{|c|}{ Sample described } & 53 & 50 & & \\
\hline 4 & \multicolumn{2}{|c|}{ Treatment described } & 76 & 3 & 24 & \\
\hline 5 & \multicolumn{6}{|c|}{ Scientific category (see below) } \\
\hline 6 & \multicolumn{2}{|c|}{ Outcome defined } & 68 & 35 & & \\
\hline 7 & \multicolumn{2}{|c|}{ Data clearly presented } & 59 & 44 & & \\
\hline 8 & \multicolumn{2}{|c|}{ Analysis required } & 66 & 37 & & \\
\hline 9 & \multicolumn{2}{|c|}{ Statistical techniques used } & 24 & 42 & & 37 \\
\hline 10 & \multicolumn{2}{|c|}{ Statistical techniques described } & 16 & 8 & & 79 \\
\hline 11 & \multicolumn{2}{|c|}{ Statistical techniques appropriate } & 11 & 6 & 7 & 79 \\
\hline 12 & \multicolumn{2}{|c|}{ Calculations correct } & 8 & 2 & 14 & 79 \\
\hline 13 & \multicolumn{2}{|c|}{ Conclusions justified } & 35 & 62 & 6 & \\
\hline 14 & \multicolumn{2}{|c|}{ Title accurate } & 95 & 8 & & \\
\hline & \multicolumn{2}{|c|}{$\begin{array}{l}\text { Mention of statistician } \\
\text { Co-author } \\
\text { Acknowledged } \\
\text { No }\end{array}$} & $\begin{array}{l}2 \\
4\end{array}$ & 97 & & \\
\hline 16 & \multicolumn{6}{|c|}{ Sample size (see below) } \\
\hline 5 & \multicolumn{3}{|c|}{$\begin{array}{l}\text { Scientific category } \\
\text { Case series } \\
\text { Case control study } \\
\text { Series with historical controls } \\
\text { Non-randomised trial with concurrent controls } \\
\text { Randomised controlled trial } \\
\text { Other }\end{array}$} & $\begin{array}{r}73 \\
3 \\
2 \\
11 \\
5 \\
6\end{array}$ & & \\
\hline 6 & Sample size & $\begin{array}{l}6-20 \\
21-40 \\
41-100 \\
101-200 \\
\text { Over } 200\end{array}$ & & $\begin{array}{r}31 \\
26 \\
27 \\
9 \\
10\end{array}$ & & \\
\hline
\end{tabular}


R. W. MORRIS

collected in a standard form, data which can be used for decision-making and later comparison. This lack of quantitative results extended even to some animal experiments in one of which, for example, tendons were merely reported to be "thicker than normal".

Subjective judgement of outcome, either by surgeon or patient, may be unavoidable but it causes problems. To classify results as acceptable or unacceptable should depend on criteria which are unequivocal for another surgeon attempting to assess a different set of similar patients; but such a rating is certain to be heavily influenced by the surgeon's enthusiasm for the treatment.

Patient's subjective opinions give similar problems. How "patient satisfaction" is graded will depend very much on the degree of happiness of both patient and surgeon as well as their attitude to each other. If patients were questioned by the surgeon who operated on them, authors should make this clear, since patients would inevitably be inhibited about reporting dissatisfaction.

Subjective opinions are important, but should be used only when two procedures are being compared. Moreover, the surgeon carrying out the operation should not be the same as the surgeon who assesses the outcome. Presentation of data. In only 59 papers were data judged to be clearly presented. The reader wants tangible results, but to present raw data on every individual case is impossible for a large series; a few crisp tables with supporting text are usually adequate. One paper employed statistical techniques without giving much actual data, and comparisons of several variables before and after operation were subjected to significance tests. This gave a veneer of statistical sophistication, but hid the raw data so that the spread of individual results around the mean values were not revealed.

Length of follow-up, or time till union of fractures were often presented in terms of means and ranges. The mean value of a series is well known to be influenced by outlying values, and the range will always be larger for greater sample sizes. The use of the median (middle value) and 10th and 90th centiles is more informative, when there is enough data to justify it (Gore and Altman 1982).

Need for statistical analysis. A small series may not require analysis, especially if individual data are given. Of the 103 papers, 66 seemed to require analysis, but only 24 had used a statistical technique. Many case series involved follow-up for widely varying lengths of time; the relationship between the outcome and the follow-up time often needs examination. A technique for analysing survival time, as in Tew and Waugh (1982) is of great value in this context.

Significance tests have been misused in medical journals (Gardner and Altman 1986). However, an apparent difference in success rates, for example, could arise from chance; significance tests are then a crude but useful tool. One paper reported the results of two methods of fixation after osteotomy but failed to compare statistically the incidence of poor results.

Unfortunately $p$-values arising from significance tests often fail to provide much useful information. A non-significant result does not prove the equivalence of two treatments; merely that the observed difference could well have arisen by chance. Small sample sizes can often yield non-significant results when in truth large differences exist.

Hence, when simple estimates of success rates are given, the use of confidence intervals is recommended, since this makes it clear that the smaller the sample the less reliable is the estimate. A paper reporting results from 288 patients with fresh fractures, and 50 with ununited fractures gave a success rate of $94 \%$ for both groups. But the $95 \%$ confidence interval for fresh fractures is $91.3 \%$ to $96.7 \%$ whereas for un-united fractures it is $87.4 \%$ to $100 \%$; on the given data, the true success rate in the smaller series could be as low as $87.4 \%$ and the reader should be made aware of this.

Use of statistical techniques. Only 16 of the 24 papers which used a statistical technique actually described it. In other papers p-values were quoted, but authors did not say how they had been calculated. In 11 of these 24 papers the statistical technique was appropriate, but in seven it was unclear, and in six it was inappropriate. In one paper significance tests designed for two samples were used when only one sample of patients was being analysed; similarly, in another paper for comparison between operated and contralateral limbs. Still other papers contained either bizarre or oversimplified statistical techniques.

One consistent problem throughout the Journal concerned the reporting of two operations on the same patient as if they were independent. A report on operations on 188 toes belonging to 62 children reported a $95 \%$ success rate (179 out of 188 toes). However, the success rate with respect to the number of children who had complete success might have been as low as $85 \%$.

The calculations based on statistical techniques were incorrect in two papers and unclear in 14. The two incorrect calculations were associated with faulty statistical techniques.

Conclusions drawn by the authors. In 62 papers, at least one of the expressed conclusions did not seem to RWM to be justified by the results. This was more likely where the aim of the paper was not clearly described. Calculation of the odds for various factors showed that fully justified conclusions were more likely to have been given if the study was not a case series, if outcome variables were properly defined and if statistical techniques were used.

The title of the paper was usually an accurate representation of its content, but in eight papers the wording was slightly misleading.

Use of statisticians. In only two papers was a statistician one of the authors, and in only four papers was a 
statistician acknowledged. This is not very surprising since many authors were not associated with medical schools. Indeed, statisticians are not always needed. They are mandatory only for large scale collaborative research; in other studies, the judicious use of statistical principles by authors themselves may be sufficient. Sample size. Over half the papers involved 40 subjects or less: the precision of estimates of success of operations in such studies is limited. If $\mathbf{3 2}$ out of $\mathbf{4 0}$ operations are deemed successful $(80 \%)$, the $95 \%$ confidence interval is $67.6 \%$ to $92.4 \%$. The accuracy of an estimate is proportional to the square root of the sample size, so to double the accuracy, the sample size must be quadrupled. Over 100 patients were reported in 19 papers, but this number is clearly impossible for many conditions. Authors should, however, be aware of and express the limitations of their results.

\section{DISCUSSION}

Differences between statisticians. A statistician's assessment of the quality of a surgical paper is as subjective as a surgeon's rating of success. Comparison of assessments were made by three statisticians on a random sample of 26 of the papers; this revealed some differences of opinion (Table III). Their views differed on sample selection, definition of outcome variables, data presentation, the need for statistical analysis and on the conclusions. It seemed that the original author (RWM) may have been too strict in his judgements on outcome variables and on the justification of conclusions.

On outcome variables, disagreement was over the

Table III. Inter-observer agreement between assessments by RWM and by two statistical colleagues, for a subsample of 26 papers

\begin{tabular}{|c|c|c|c|c|}
\hline \multirow{2}{*}{\multicolumn{2}{|c|}{ Item }} & \multicolumn{3}{|c|}{$\begin{array}{l}\text { Number of papers for which "yes" } \\
\text { was recorded }\end{array}$} \\
\hline & & $\mathbf{R W M}$ & Colleague $A$ & A Colleague B \\
\hline 1 & Aim described & 24 & 24 & 26 \\
\hline 2 & Population defined & 23 & 22 & 25 \\
\hline 3 & Sample described & 15 & 16 & 10 \\
\hline 4 & Treatment described & 18 & 18 & 18 \\
\hline 5 & Study category - case series & 20 & 19 & 23 \\
\hline 6 & Outcome defined & 15 & 21 & 22 \\
\hline 7 & Data clearly presented & 14 & 11 & 21 \\
\hline 8 & Analysis required & 14 & 15 & 22 \\
\hline 9 & Statistical techniques used & 6 & 8 & 9 \\
\hline 10 & Statistical techniques described & 4 & 4 & 4 \\
\hline 11 & Statistical techniques appropriate & 2 & 2 & 2 \\
\hline 12 & Calculations correct & 1 & 0 & 1 \\
\hline 13 & Conclusions justified & 5 & 15 & 22 \\
\hline 14 & Title accurate & 25 & 24 & 24 \\
\hline
\end{tabular}

value of a subjective but fairly well defined assessment by surgeon or patient, and RWM's criticism was based on the lack of comparable control data. If it is accepted that control data may be impossible to collect then this criticism is less relevant.

On conclusions, RWM's strict response in this highly subjective area should probably only be seen as a reflection of his dissatisfaction with other aspects of the papers, but even on reassessment of the 26 papers, he still judged that three quarters included at least one unjustified conclusion.

\section{RECOMMENDATIONS}

Specific improvements. The method of selecting patients should be described in more detail (this was sometimes totally lacking), outcome variables should be properly defined, data should be presented more clearly and the use and description of statistical techniques should be enhanced.

Conclusions should not be stated, especially in the abstract, unless they are completely justified by the results presented. Conclusions which are printed in abstracts tend to be enshrined as truth; authors have a large responsibility in this respect. Advocacy of a given operation, when it is based on apparent success in a small case series, should be appropriately cautious.

The use of statistical referees has increased in recent years, with a consequent improvement in the statistical quality of some journals. Many papers contain the potential for useful information, if some of the points outlined above had been followed more closely. A useful text is provided by Altman et al. (1983). Textbooks which expound statistical principles more generally include Maxwell (1978) and Bradford Hill (1984), while both Armitage (1971) and Colton (1974) discuss more advanced techniques.

General improvements. Many orthopaedic papers contain case series without controls, and often report small numbers of subjects. Many disjointed pieces of research, often retrospective, are submitted to the British Volume of The Journal of Bone and Joint Surgery. Accepting the hierarchy of scientific evidence (Green and Byar 1984), these reports are unlikely to provide convincing arguments for or against alternative procedures though they may well provide useful information - for example about new tests or signs. Surgeons must therefore follow their own instincts in patient management rather than be guided by carefully conducted research. The exaggerated claims made in some papers may be misleading.

For rare conditions or complications substantial sample sizes are not available to a single surgeon. If, in addition, long-term follow-up is required, an objective report is impossible. The answer lies in multi-centre collaborative research, involving specific protocols on patient inclusion, treatment, evaluation of outcome and conduct of follow-up. Such specific research would be 
most useful when there is genuine doubt as to which of two or more procedures is to be recommended for a relatively common condition.

Often, reports of large series followed for an adequate time are not comparable with other reports in the literature because of a different patient mix, different types of postoperative care and other problems. The usual recommendation is to conduct a double-blind randomised controlled trial, although this option is regarded ambivalently by surgeons (Dudley 1985). Special approaches are needed for such studies, but randomised trials are still to be encouraged (Lancet 1986). Large multi-centre trials will require the collaboration of a statistician in designing the study and in calculating the necessary sample size (most sample sizes were inadequate in $J$ Bone Joint Surg $[\mathrm{Br}]$ 1984). He should also advise in conducting the study, especially with respect to random allocation of patients to treatments and to the accumulation of data, as well as helping to conduct the analysis. Even small scale research papers would benefit from greater use of statistical principles and techniques. This will be fostered by seeking the advice of statisticians at all stages of any quantitative study. Pocock (1983) provides a comprehensive practical guide to the conduct of clinical trials.

Writing a research paper. The checklist in Table I may be used in review; but at the outset the following questions are useful:

1 - What question are we trying to answer?

2 - To what population or group do our findings apply?

3 - What criteria are we using to assess success? (What is the outcome variable?) Can it be measured in a standardised way for all subjects.?

4 - Is statistical analysis necessary, and if so, what techniques should we use?
5 - What conclusion may we legitimately make, after reconsidering the type of study, and the sample size?

I should like to thank Mr A. Graham Apley, Mr Philip Fulford and Professor W. W. Holland for encouraging me to carry out this project: Miss S. Chinn and Mrs S. Patel for their help with statistical assessments, and Miss A. Godfrey for typing this manuscript. RWM is supported by St Thomas' Hospital Special Trustees.

\section{REFERENCES}

Altman DG, Gore SM, Gardner MJ, Pocock SJ. Statistical guidelines for contributors to medical journals. Br Med J 1983;286:1489-93.

Armitage P. Statistical methods in medical research. Oxford and Edinburgh: Blackwell Scientific, 1971.

Bland JM, Jones DR, Bennett S, Cook DG, Haines AP, Macfarlane AJ. Is the clinical trial evidence about new drugs statistically adequate? Br J Clin Pharmacol 1985;19:155-60.

Colton T. Statistics in medicine. Boston: Little, Brown and Company, 1974.

Dudley HAF. Trials and tribulations for surgeons. Br J Surg 1985;72:255.

Evans M, Pollock AV. A score system for evaluating random control clinical trials of prophylaxis of abdominal surgical wound infection. Br J Surg 1985;72:256-60.

Gardner MJ, Altman DG. Confidence intervals rather than $P$ values: estimation rather than hypothesis testing. $B r \quad J M e d$ 1986;292:746-50.

Gardner MJ, Machin D, Campbell MJ. Use of checklists in assessing the statistical content of medical studies. $\mathrm{Br}$ Med $J$ $1986 ; 292: 810-2$.

Gore SM, Altman DG. Statistics in practice. London: British Medical Association, 1982.

Green SB, Byar DP. Using observational data from registries to compare treatments: the fallacy of omnimetrics. Stat Med 1984;3:361-73.

Hill AB. A short textbook of medical statistics. 11 th ed. London, etc: Hodder and Stoughton, 1984.

Lancet. The epistemology of surgery. Lancet 1986; ; :656-7.

Maxwell AE. Basic statistics for medical and social science students. London: Chapman and Hall, 1978.

Pocock SJ. Clinical trials: a practical approach. Chichester, etc: John Wiley \& Sons, 1983.

Tew M, Waugh W. Estimating the survival time of knee replacements. $J$ Bone Joint Surg [Br] 1982;64-B:579-82. 